\title{
Case Report \\ Delayed Pneumomediastinum and Subcutaneous Emphysema in Covid-19 \\ Patients: Two Case Reports
}

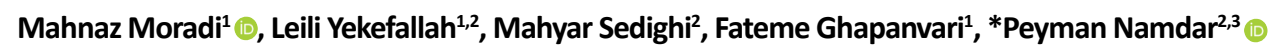

1. Department of Critical Care Nursing, School of Nursing and Midwifery, Qazvin University of Medical Sciences, Qazvin, Iran.

2. Metabolic Diseases Research Center, Research Institute for Prevention of Non-Communicable Diseases, Qazvin University of Medical Sciences, Qazvin, Iran.

3. Department of Emergency Medicine, School of Medicine, Qazvin University of Medical Sciences, Qazvin, Iran.

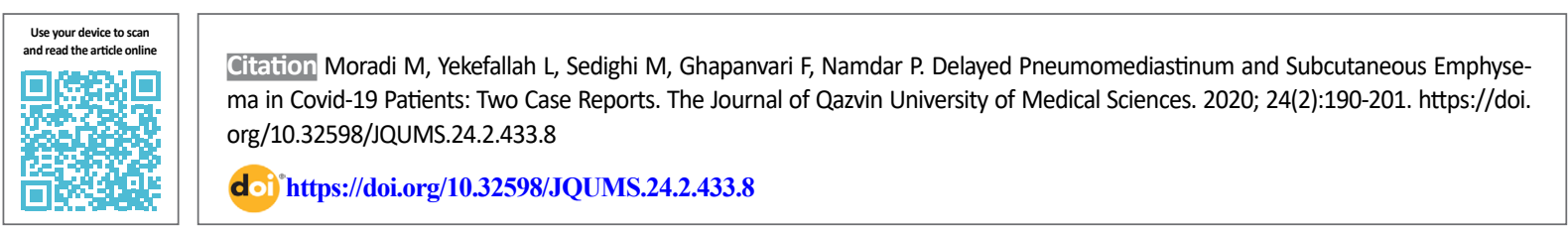

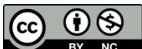

Received: 29 Mar 2020

Accepted: 04 May 2020

Available Online: 01 Jun 2020

Keywords:

Coronavirus disease

2019, Spontaneous pneumomediastinum, Subcutaneous emphysema, Pneumothorax

\section{A B STRACT}

Coronavirus Disease 2019 (COVID-19) is a severe respiratory disease initiated on December 2019. Genome sequence analysis shows that the pathogen is a complex beta-coronavirus RNA, known as Severe Acute Respiratory Syndrome Coronavirus-2 (SARS-CoV-2). Spontaneous Pneumomediastinum and pneumothorax are rare clinical and radiological conditions associated with COVID-19. This study is case report of pneumomediastinum and subcutaneous emphysema in two patients with COVID-19, while investigating the clinical and paraclinical symptoms and the treatment process of two cases. Finding factors related to the severity of COVID-19 for improving the final outcome in patients is very important.

\section{Extended Abstract}

\section{Introduction}

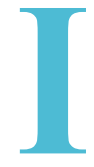

$\mathrm{n}$ the spring of 2020 , the coronavirus disease 2019 (COVID-19) outbreak spread to more than 200 countries in the world. This disease is now a serious threat to global health and continues to challenge healthcare systems around the world [1]. The epidemiological and clinical features of this disease have been under investigation since the initial identification of the virus [6].
Spontaneous pneumomediastinum is a rare clinical disease defined as the presence of free air in mediastinal structures without apparent cause such as trauma [11]. This clinical condition may be due to leakage of air from ruptured wall of marginal pulmonal alveoli, secondary to the high intra-alveolar pressure caused by factors such as artificial ventilation or a severe cough. [12]. In the present study, we report two cases of pneumomediastinum and delayed subcutaneous emphysema in COVID-19 patients.

\section{* Corresponding Author:}

Peyman Namdar

Address: Department of Emergency Medicine, Qazvin University of Medical Science, Qazvin, Iran.

Tel: +98 (28) 33336001

E-Mail: drpeymannamdar@gmail.com 


\section{Case Report}

The patient 1 was a 33-year-old man with no history of underlying disease admitted to the emergency department of Bu Ali Sina Hospital following the symptoms of shortness of breath, dry cough and tachypnea (26 times per min). After pulse oximetry reporting $80 \%$ oxygen saturation, oxygen was given to him with a bag-valve-mask and then a Bilevel Positive Airway Pressure (BIPAP) was installed in two levels of 5 and $10 \mathrm{~cm} \mathrm{H2O}$. PCR test was positive, and the CT scan showed severe bilateral ground-glass opacities in both lungs. Following the progression of hypoxia and decreased oxygen saturation, he was transferred to the ICU one day after admission. On the second day of admission, following the onset of Paroxysmal Supraventricular Tachycardia (PSVT) and having severe hypoxia and a decrease in oxygen saturation to $60 \%$, the patient was intubated and connected to the ventilator.

Despite receiving high doses of propofol, midazolam, fentanyl, and atracurium, the patient could not tolerate the ventilator, which resulted in re-extubation and re-intubation by the anesthesia resident. During the hospitalization period, the patient was treated with hydroxychloroquine, Kaletra and then atazanavir for 5 days and 7 doses of 44 mcg interferon beta. Following improvement of respiratory function, the patient's endotracheal tube was removed on the 14th day of hospitalization. Symptoms of subcutaneous emphysema appeared two days after removal. Chest CT scan reported severe pneumomediastinum and extensive subcutaneous emphysema at the base of the neck and subcutaneous area of the chest. The patient underwent conservative treatment for emphysema but died after 22 days.

The second patient was a 40-year-old man with no history of underlying disease admitted to the emergency department following fever and chills, tachypnea and dry cough that had started 6 days ago, and then transferred to the ICU next day. The PCR test was positive and the CT scan findings were in favor of Covid-19. bilateral ground-glass opacity was seen in more than $50 \%$ of the lungs. The patient initially received oxygen with a bag-valve-mask, but two days after admission to the ICU for cardiac monitoring, multiple premature ventricular contractions appeared. According to cardiac counseling, premature ventricular contractions were justified, and the patient was intubated with exacerbation of respiratory symptoms and a decrease in oxygen saturation to $75 \%$. He had no previous history of diabetes but developed it after having Covid-19.

Hence, he was prescribed NPH and regular insulin injection. During hospitalization, he was treated for 3 days with Keltra twice daily and then intravenous immunoglobulin for 3 days at a dose of $30 \mathrm{~g}$. Then, they received 7 doses of $44 \mathrm{mcg}$ interferon beta every other day. The patient's endotracheal tube was removed after 9 days when respiratory function was improved. One day after removal, signs of subcutaneous emphysema appeared with a size of 5 by $5 \mathrm{~cm}$ above the sternum which cured without surgical and invasive treatment using maintenance treatment within 3 days. Finally, after 25 days, he was discharged in a good general condition.

\section{Discussion}

To date, published studies on Covid-19 have rarely discussed the presence of delayed pneumomediastinum and subcutaneous emphysema following mechanical ventilation. In both study cases, CT scan and clinical findings showed these complications [13]. According to studies, pneumomediastinum and pneumothorax in patients with Covid-19 are complications of endotracheal intubation or mechanical ventilation in patients with chronic obstructive pulmonary disease who required aggressive ventilation to correct hypoxemia [24]. Absence of smoking history is also a common feature in most of the described cases of pneumomediastinum and pneumothorax following Covid-19 [21]. These findings are consistent with the present study, because neither of the two patients had a history of smoking or chronic obstructive pulmonary disease. Probably the main cause of pneumomediastinum and subsequent subcutaneous emphysema was Covid-19 and its destructive effect on the alveoli. Patients diagnosed with Covid 19, who are being treated by non-invasive and invasive forms of ventilation, require close monitoring for complications such as pneumomediastinum and subcutaneous emphysema.

\section{Ethical Considerations}

\section{Compliance with ethical guidelines}

All ethical principles are considered in this article. This study was approved by the Ethics Committee of Qazvin University of Medical Sciences (Code: IR.QUMS. REC.1399.192).

\section{Funding}

This research did not receive any specific grant from funding agencies in the public, commercial, or non-profit sectors.

\section{Authors' contributions}

Writing - original draft and writing - review \& editing: Mahnaz Moradi, Fateme Ghapanvari, Mahyar Sedighi, and 
Peyman Namdar; Data collection: Mahnaz Moradi; Supervision: Leili Yekefallah, Mahnaz Moradi.

Conflicts of interest

The authors declared no conflict of interest. 


\title{
ظهور بنومومدياستن و آمفيزم زيرجلدى تأخيرى در كوويد ــ 19: تزارش دو مورد
}

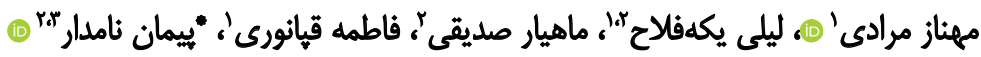

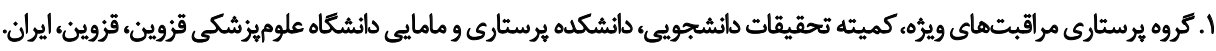

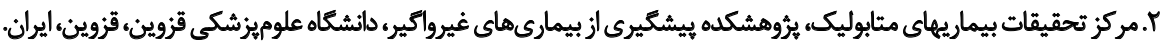

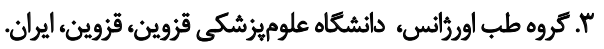

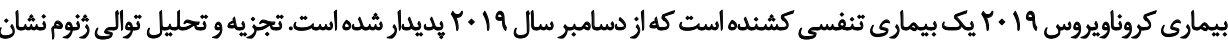

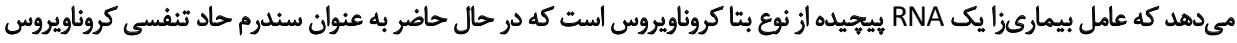

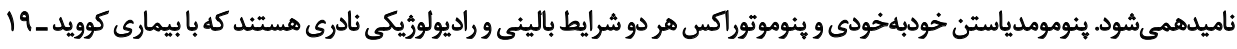

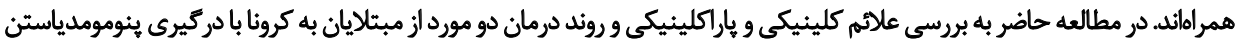

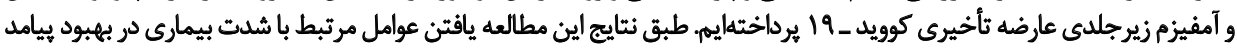

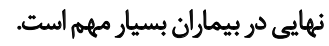

تاريخ دريافت: •ا فروردين

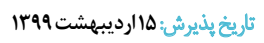
تاريخ ايتشار: rاخرداد

: بيمارى كروناويروس

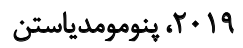

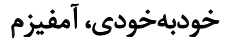
زيرجلدى، ينوموتوراكس، آمغيزم

تنفسى فوقانى (نظير آبريزش بينى، عطسه، يا كلودرد) را نشان

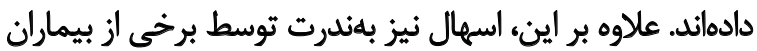

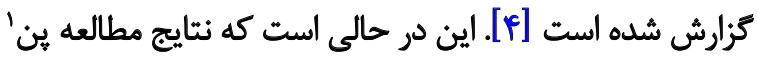

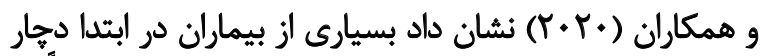

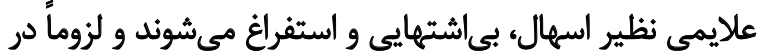

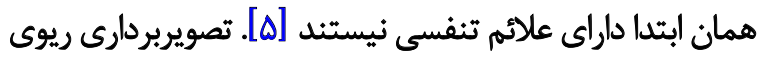

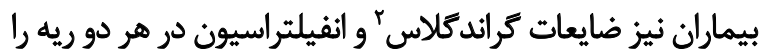

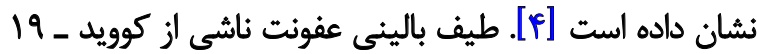

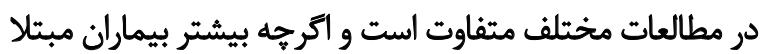

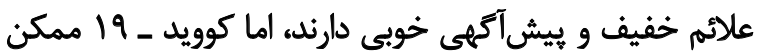

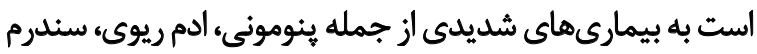

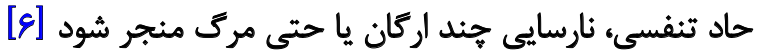

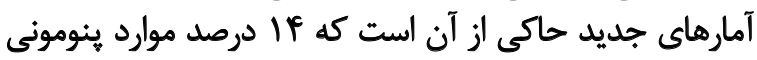

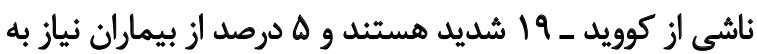

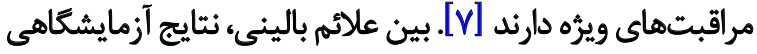

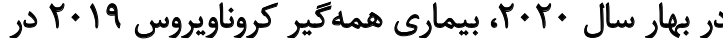

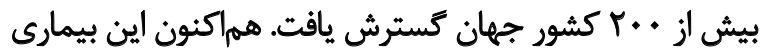

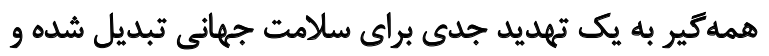

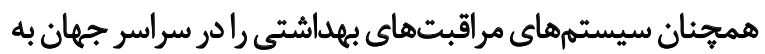

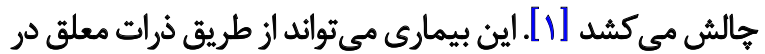

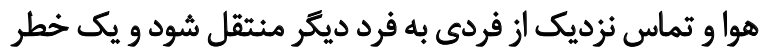

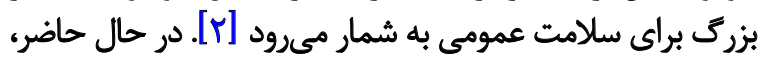

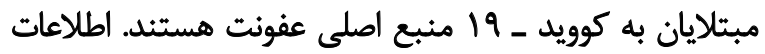

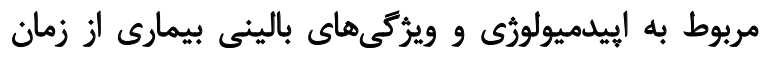

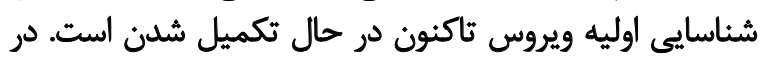

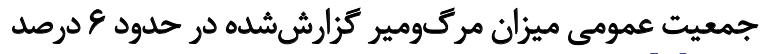

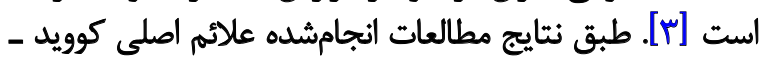

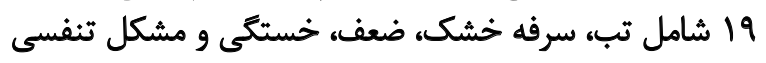

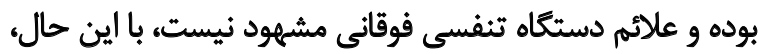
تعداد معدودى از بيماران مبتلا به كوويد ـ 9 اله علائم دستكاه

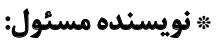
بيمان نامدار

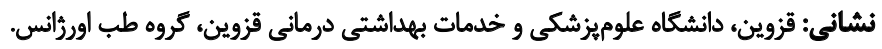

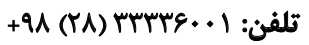
راياثامه: drpeymannamdar@gmail.com 
آلوئل مى تواند با فشار بالاى داخل آلوئلى ناشى از عواملى مانند

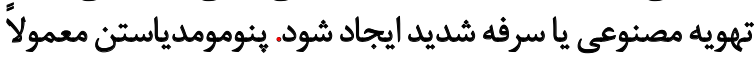

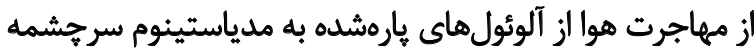

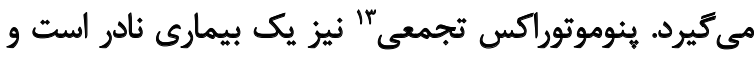

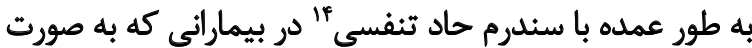

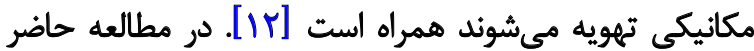

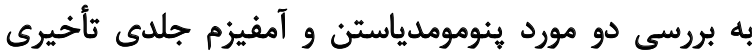
مي يردازيم.

\section{معرفي بيمار 1}

بيمار آقاى بساسالهاي بود كه سابقه هيج كونه بيمارى زمينهاي

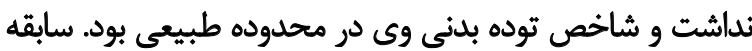

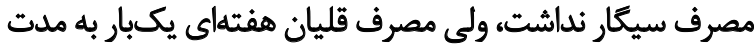

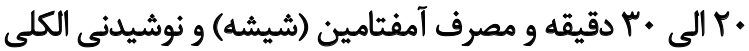

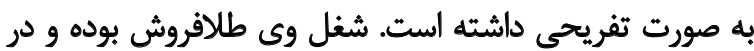

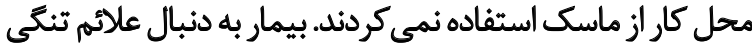

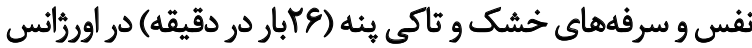

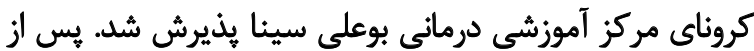

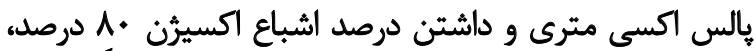

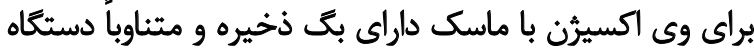

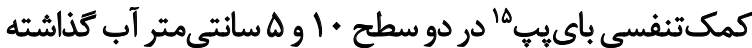

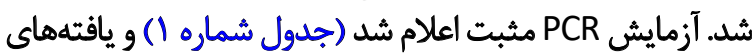

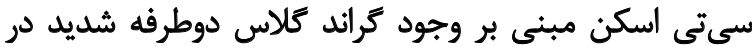

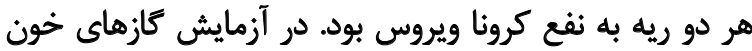

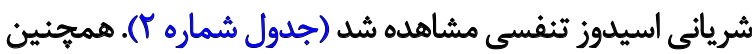

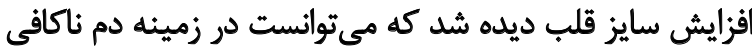

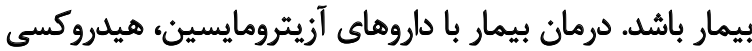

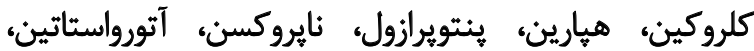

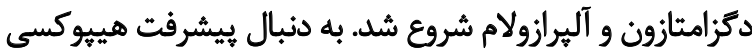

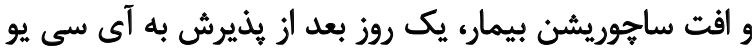

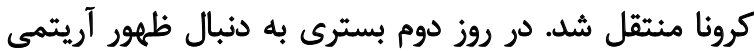

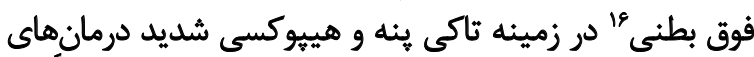

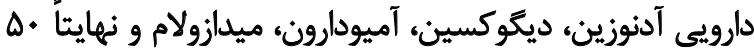

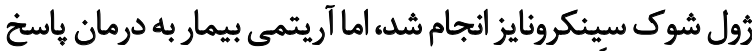

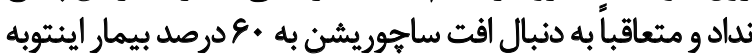

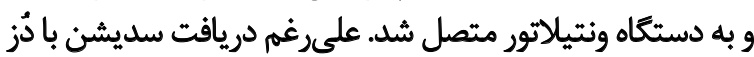

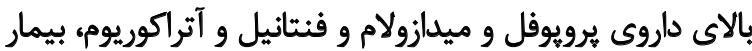

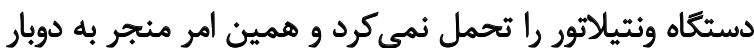

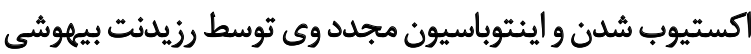

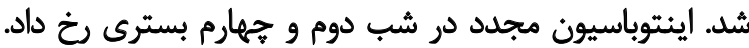

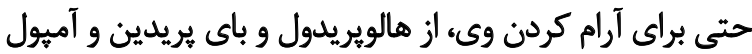

13. Loculated Pneumothorax (LPNX)

14. Acute Distress Respiratory Syndrome (ARDS)

15. Bilevel Positive Airway Pressure (BIPAP)

16. Paroxysmal Supraventricular Tachycardia (PSVT)
و تظاهرات سى تى اسكن ريه، در مبتلايان به نوع خفيف و

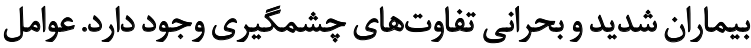

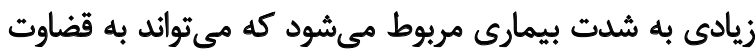

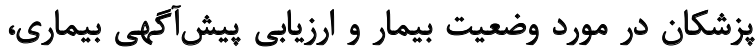

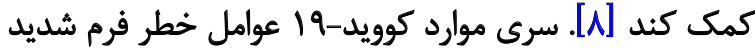

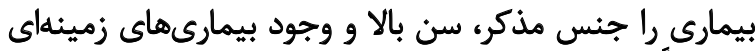

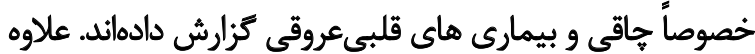

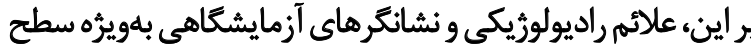

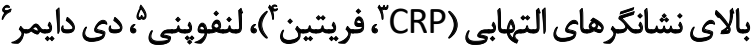

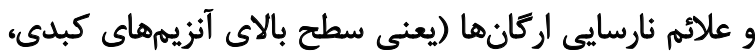

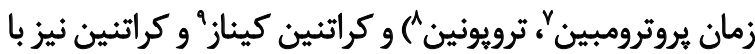

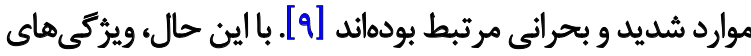

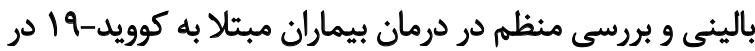

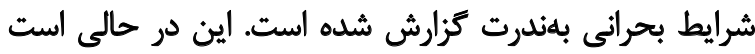

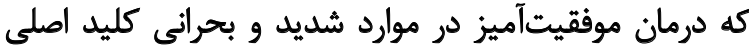

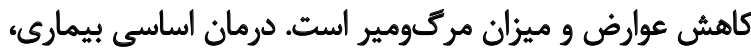

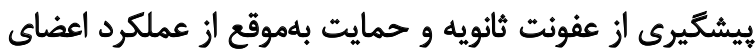

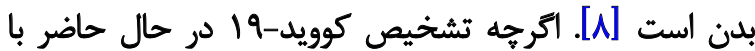

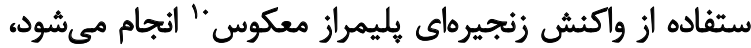

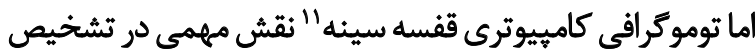

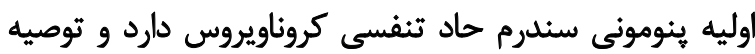

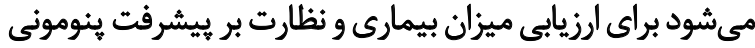

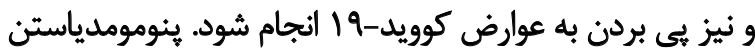

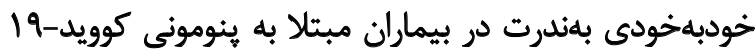

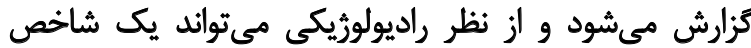

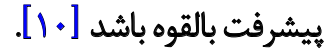

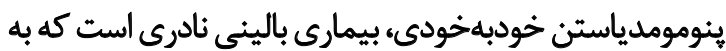

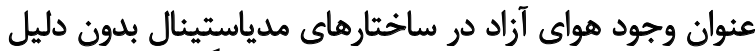

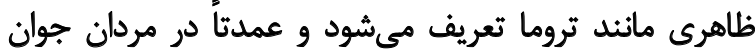

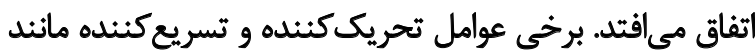

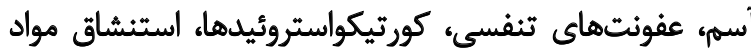

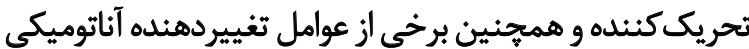

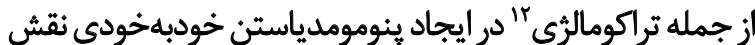

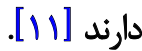

اين وضعيت بالينى ممكن است در اثر نشت هوا از ديواره آلوئلهاى يارهشده به فضاى مدياستن ايجاد شود. آسيب ديواره
3. C-reactive protein
4. Ferritin
5. Lymphopaenia
6. D-dimer
7. Prothrombin Time (PTT)
8. Troponin
9. Creatine Phosphokinase (CPK)
10. Reverse Transcriptase Polymerase Chain Reaction (RT-PCR)
11. Chest Computed Tomography (CT)
12. Tracheomalacia 
به بيمارستانى ديكر در شهر تهران (خصوصى) جهت ادامه روند

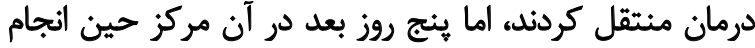
يروسيجر توراكوتومى فوت شدند.

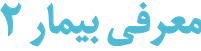

بيمار آقاى • fاساله بدون هيج سابقهاى از بيمارى زمينهاى و

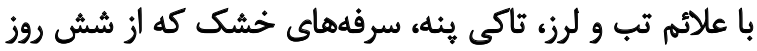

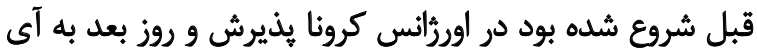

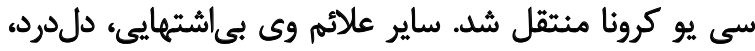

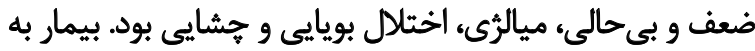

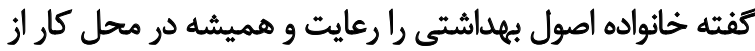

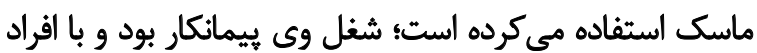

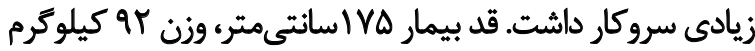

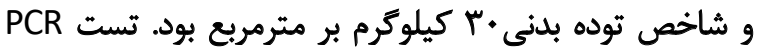

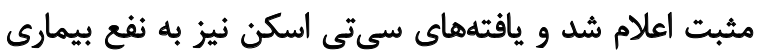

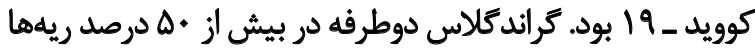
مشهود بود (شكل شماره T).

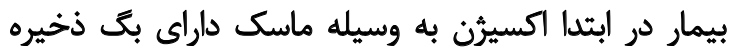

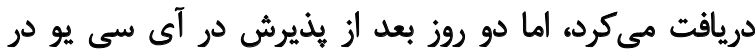

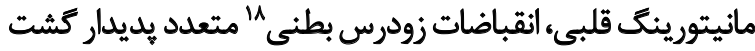

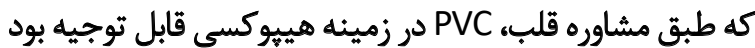

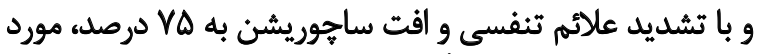

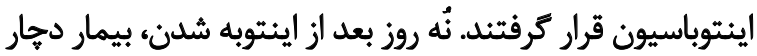

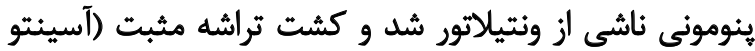

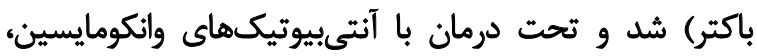

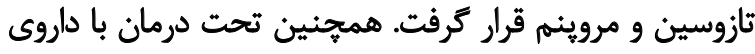

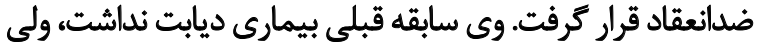

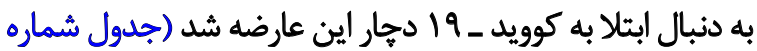

18. Premature Ventricular Contraction (PVC)

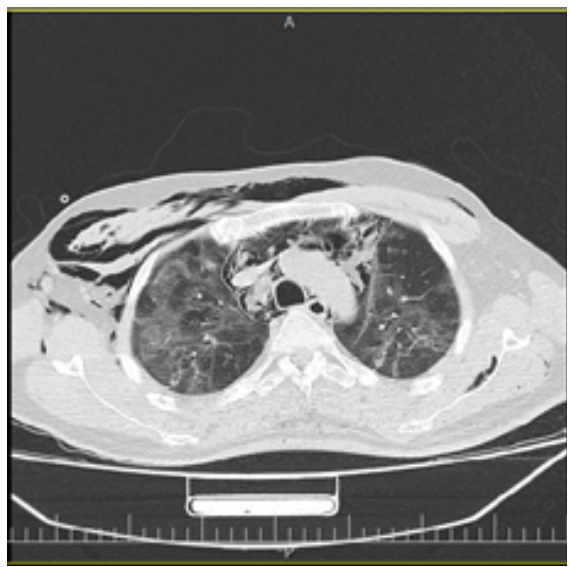

متادون (احتمالاً به دليل سابقه مصرف آمفتامين) نيز استفاده

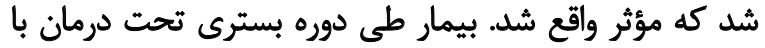

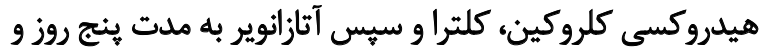

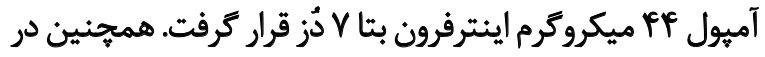

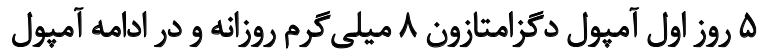

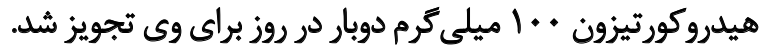

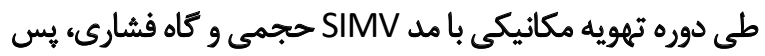

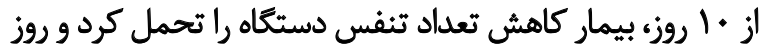

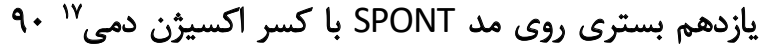

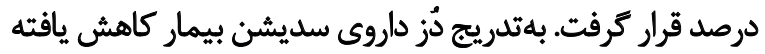

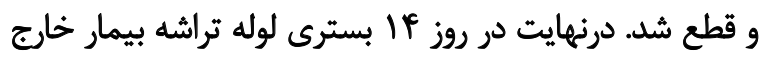

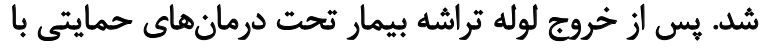

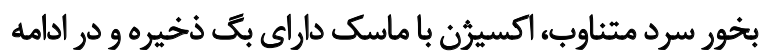

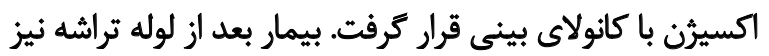

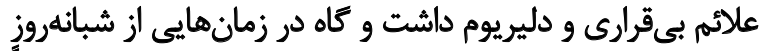

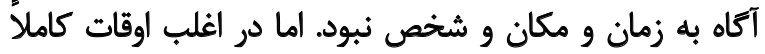

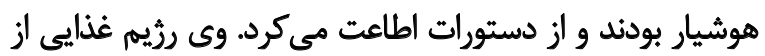

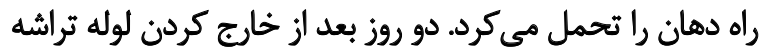

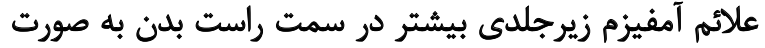

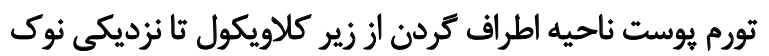

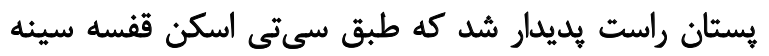

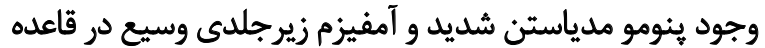

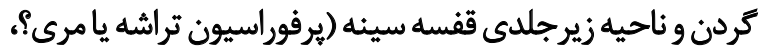

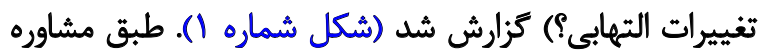

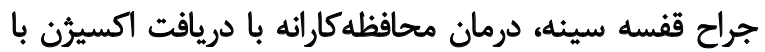

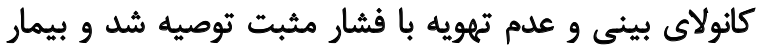

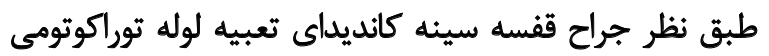

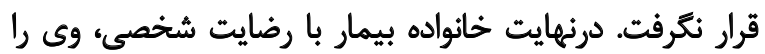

\section{Fraction of Inspired Oxygen (FIO2)}

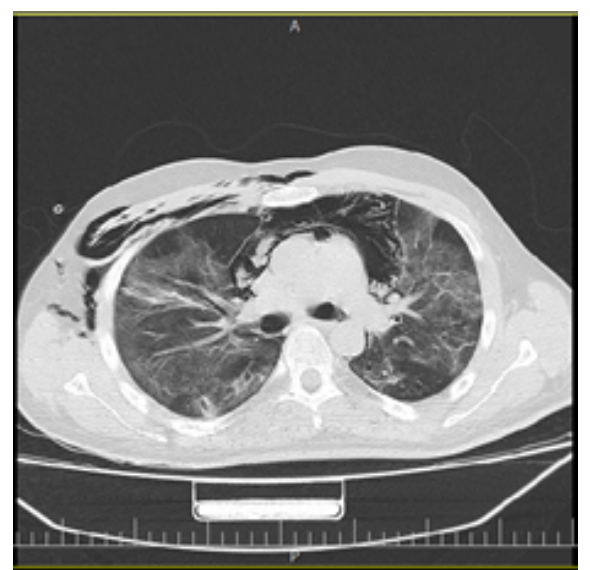


جدول ا. نتيجه آزمايشات دو بيمار

\begin{tabular}{|c|c|c|c|c|c|c|c|c|}
\hline \multirow{2}{*}{ مقادير نرمال } & \multicolumn{3}{|c|}{ بيمار r } & \multicolumn{3}{|c|}{ بيمار 1} & \multirow{2}{*}{ واحد } & \multirow{2}{*}{ اززمايشات } \\
\hline & ترخيص & اواسط بسترى & روز اول & ترخيص & اواسط بسترى & روز اول & & \\
\hline$p-1$. & 1.10 & $\sqrt{ } /$ & $1 r / 9$ & $9 / r$ & $1 . / \Delta$ & $N r$ & .... واحد در ليتر & كلبول سفيد \\
\hline$r / r-\Delta / r$ & $F / / F$ & $F / M$ & $\Delta / T \gamma$ & $F / M$ & $F / T$ & $F / N$ & ه... واحلد در ليتر & كلبول قرمز \\
\hline$\| r-18$ & $\mathbb{H / S}$ & $\mathbb{N} / \pi$ & 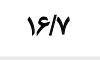 & $I T / N$ & $I F /$ & $10 / 9$ & كرم/دسىليثر & هموكلويين \\
\hline$r \Delta / q-\mu \varphi / 8$ & $r e / r$ & $\pi / 9$ & pV/s & $F+11$ & FNY & $r A / A$ & درصد & هماتوكريت \\
\hline intra. & MTr & q. & $1+9$ & $18 v$ & las & mII & •... واحد در ليتر & بالاكت \\
\hline$r+-r+$ & 18 & $r$ & 8 & 9 & r & 8 & ل درصد & لنفوسيث \\
\hline$P A-P q$ & $\mathrm{VA}$ & qp & qr & A) & ar & $M$ & درصد & نوتروفيل \\
\hline$v \cdot-11$. & iar & mif & $1 . r$ & - & - & $M$ & ميلى كرم/دسىليتر & ق قندخون ناشتا \\
\hline$r *>$ & - & - & m & - & $n$ & - & ميلىمتر & ميزان سليمان الريتروسيت \\
\hline$Y-r)$ & rq & FA & r. & $m$ & ג & ro & ميلى كرم / دسى ليتر & نيتيروزن اوره خون \\
\hline$\cdot \mid 8-1 /$ &.$/ 9$ & r & $1 / \Delta$ &.$/ 9$ & 1 & 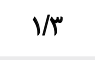 & ميلى كرم/دسىليتر & كرائين \\
\hline Irg-IFa & ו & Ira & IIT & if. & ir. & ife & ميلى اكى والان /ليتر & سديم \\
\hline$r / 8-\Delta / 1$ & $r$ & $p / 8$ & $\Delta / 1$ & $r / h$ & $\Delta / V$ & $\Delta / \Delta$ & ميلى آلكى والان اليتر & يثاسيم \\
\hline$N \Delta-1.1 \Delta$ & Ne & $\sqrt{ } /$ & Ne & NA & $V / 8$ & Na & ميلى كرم/دسى ليتر & كلسيم \\
\hline$T / \Delta-\Delta$ & $r / \Delta$ & - & r & $M M$ & $r / A$ & $r / r$ & ميلى كرم/دسىليتر & فسفر \\
\hline $1 / 8-r / 0$ & $r / P$ & $r / \lambda$ & $r / A$ & r & $T / N$ & $r / \Delta$ & ميلى كرم/دسى ليتر & منيزيم \\
\hline$r-I^{f}$ & $i f / r$ & $r e / q$ & $1 r / \Delta$ & $I f / N$ & $\| W / \Delta$ & 191 & ثاثيه & زمان يروترومبين \\
\hline 1 & V/r & 1/99 & 1 & WV & $y \cdot A$ & $1 / T A$ & ثاثيه & $\begin{array}{c}\text { نسبت نرمال يين|لملإلى } \\
\text { International Normalized } \\
\text { Ratio (INR) }\end{array}$ \\
\hline
\end{tabular}

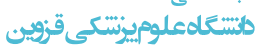
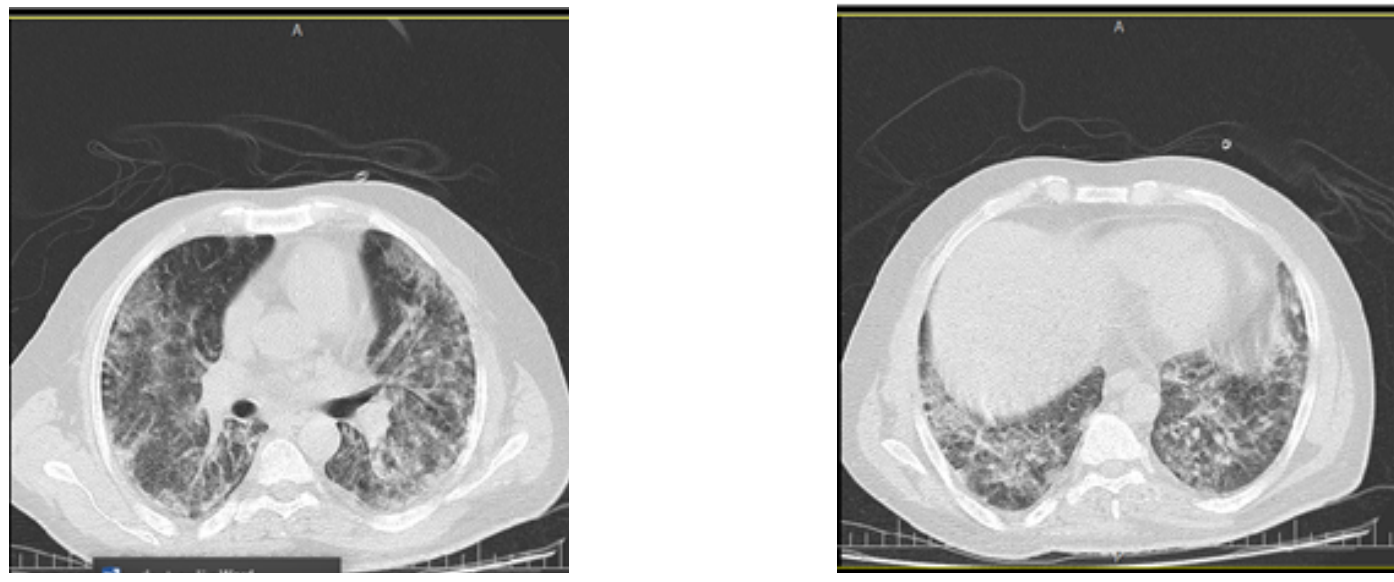

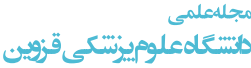

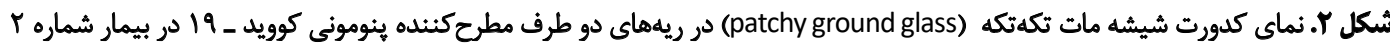

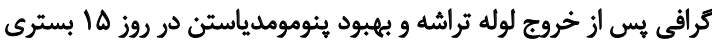


جدول ب. نتيجه كازهاى خون شريانى و وريدى دو بيمار

\begin{tabular}{ccccccc}
\hline & & & & \\
\hline \\
\hline
\end{tabular}

\section{مانتستاهعلوميزنتكى قزوين}

1. Potential Hydrogen (PH)

2. pressure of carbon dioxide $\left(\mathrm{PaCO}_{2}\right)$

3. Partial Pressure of Oxygen $\left(\mathrm{PaO}_{2}\right)$

داشتند. دليريوم بيمار باستفاده از حضور همر اهان در بالين بيمار

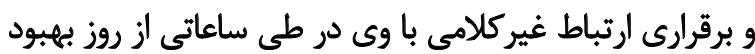

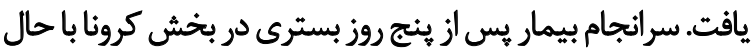

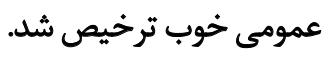

\section{بحث و نتيّهاه كَيرى}

تا به امروز، در مطالعات منتشرشده در خصوص بيمارى كوويد

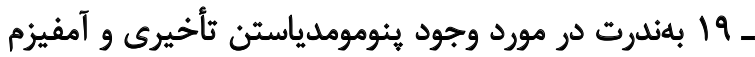

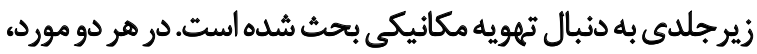

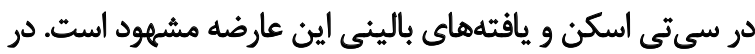

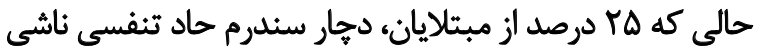

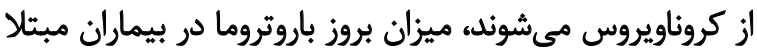

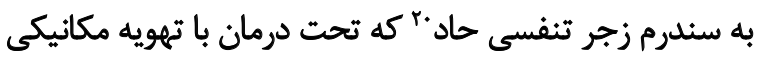

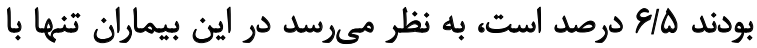

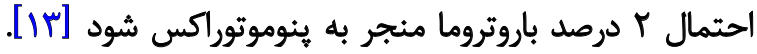

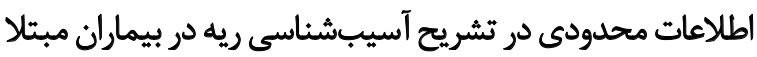

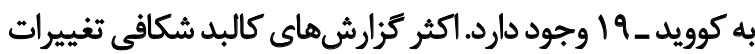

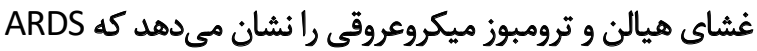

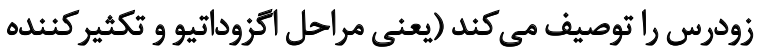

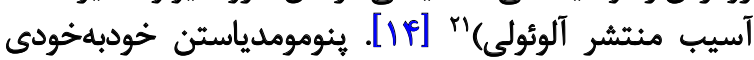
يك بيمارى نادر و به طور كلى خوشخيم است كه به صورت
4. Serum Bicarbonate ( $\mathrm{HCO} 3)$

5. Saturation of Peripheral Oxygen $\left(\mathrm{SPO}_{2}\right)$

6. Base Excess (BE)

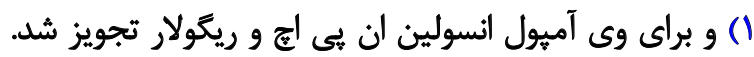

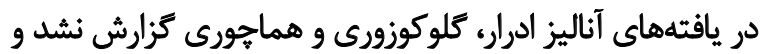

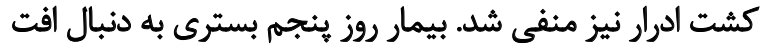

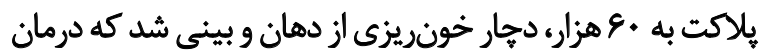

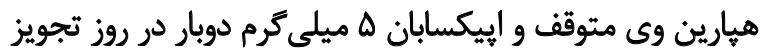

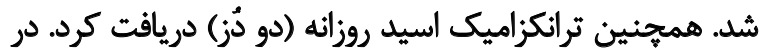

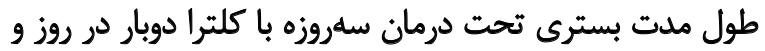

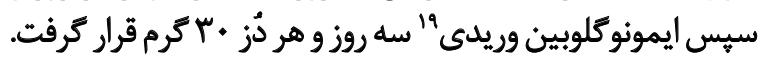

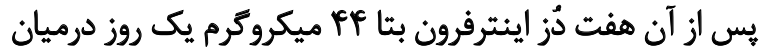

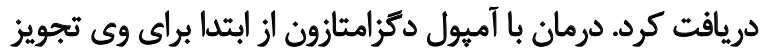

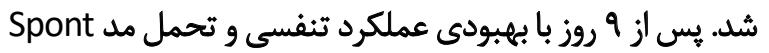

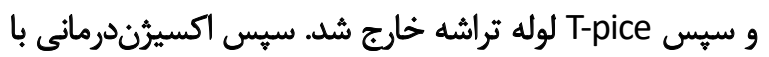

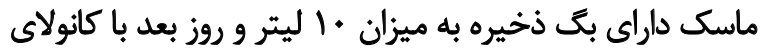

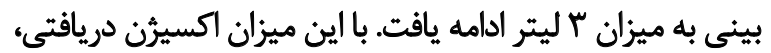

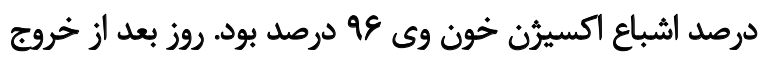

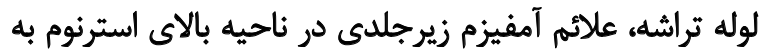

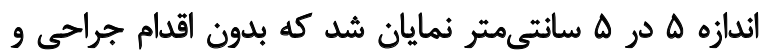

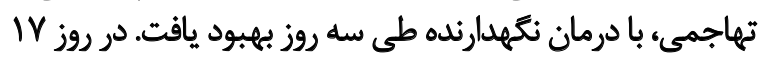

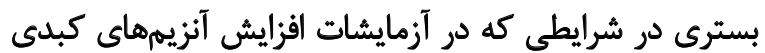

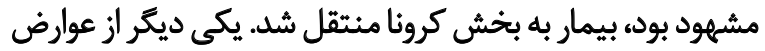

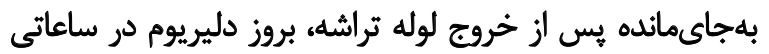

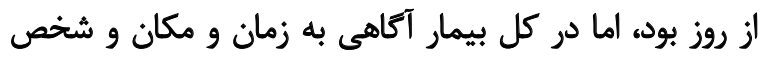


آسيبرساننده ميزبان كه معمولاً در ينومونى ويروسى مشاهده

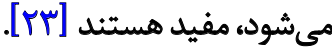

ينومومدياستن و ينوموتوراكس در مبتلايان به كوويد ــ 19 ازئاز

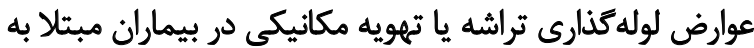

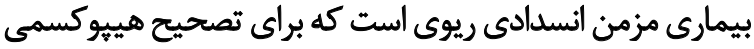

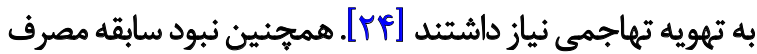

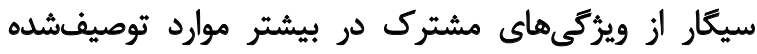

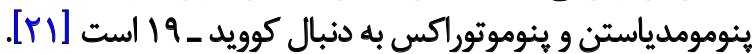

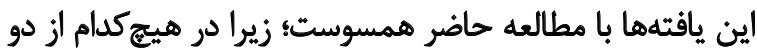

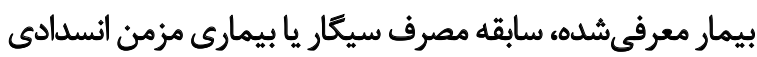

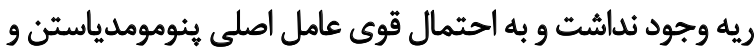

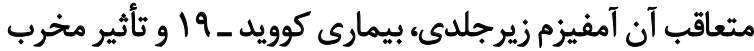

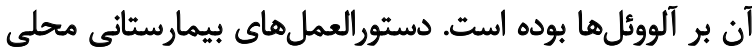

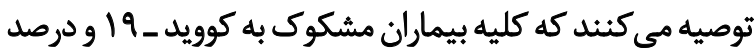

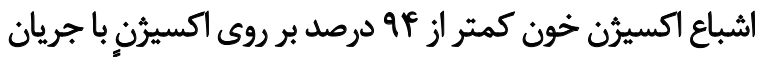

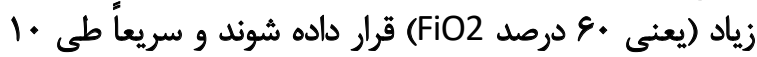

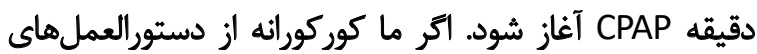

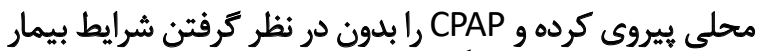

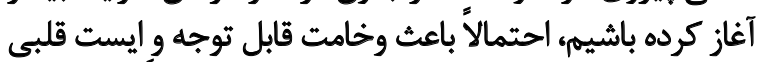

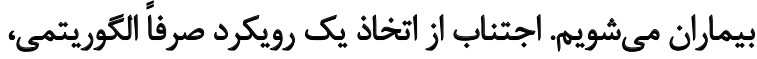

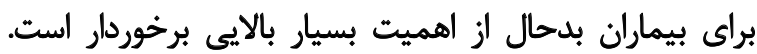

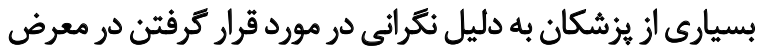

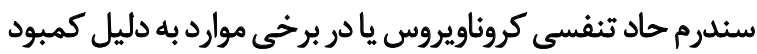

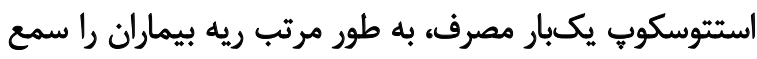

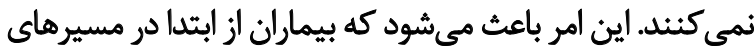

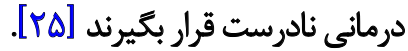

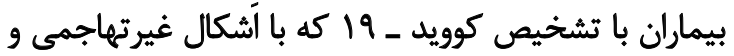

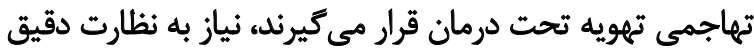

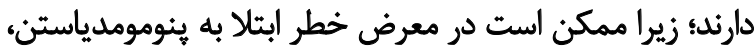

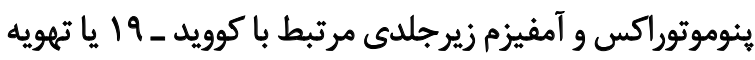

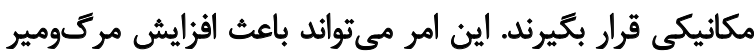

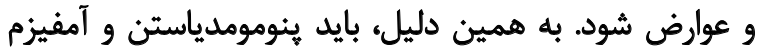

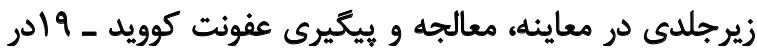

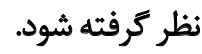

\section{مالاحظاث اخغالاقي \\ ييروى از اصول اخلاق يروهش}

اين مطالعه در دانشكاه علوميزشكى قزوين با شناسه اخلاق

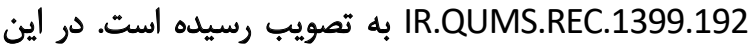

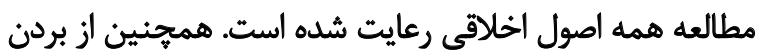

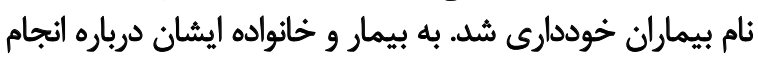

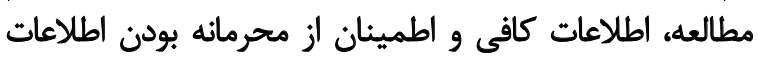

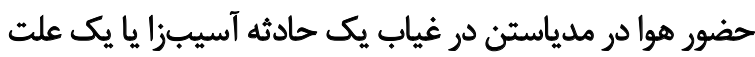

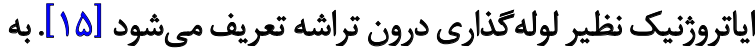

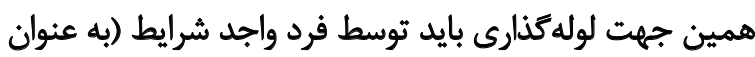

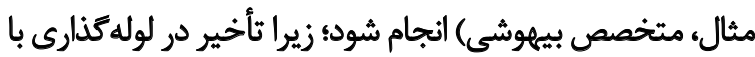

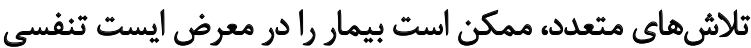

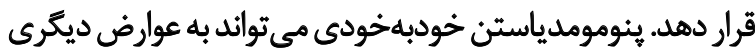

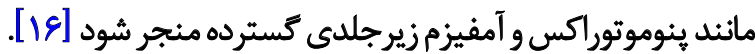

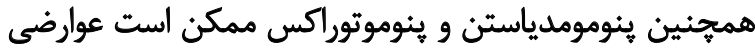

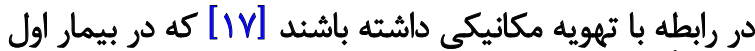

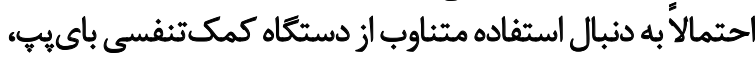

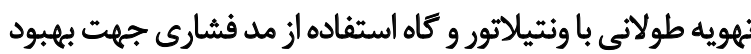

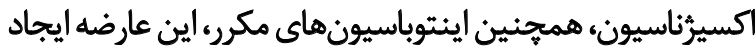

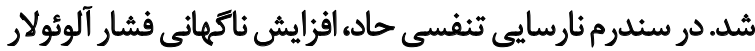

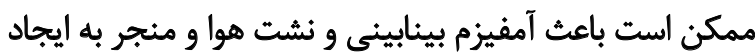
آمفيزم مدياستين شود [1/]].

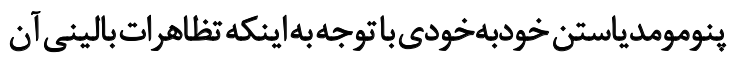

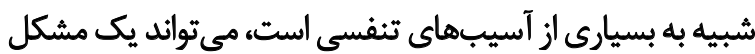

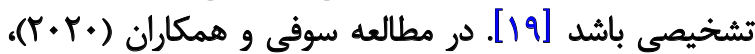

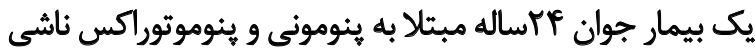

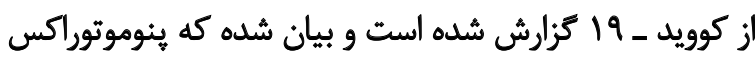

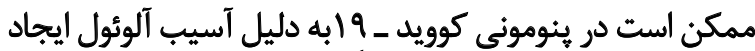

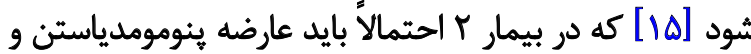

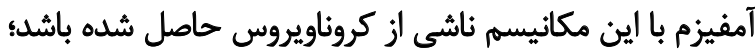

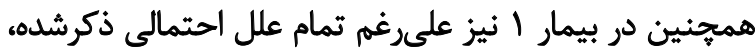

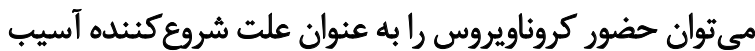

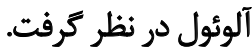

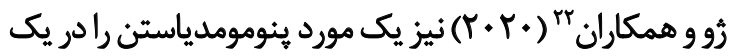

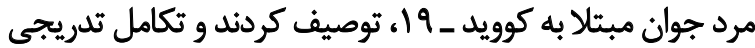

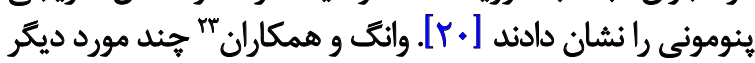

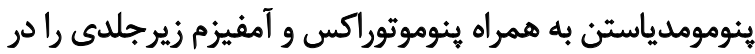

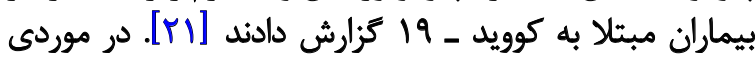

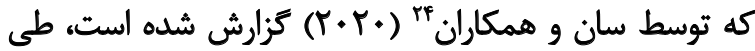

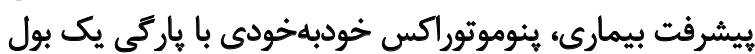

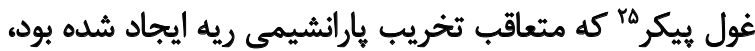

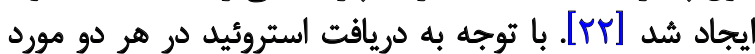

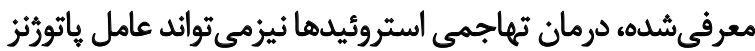

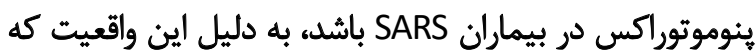

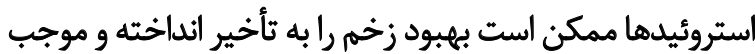

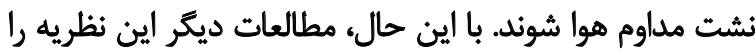

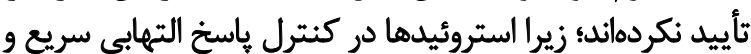

\section{Zhou}

23. Wang

24. Sun

25. giant bulla 


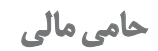

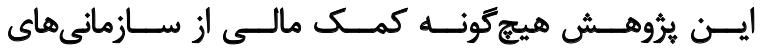

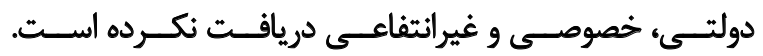

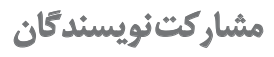

جستوجوى مقالات: مهناز مرادى و فاطمه قيانورى؛ كردآ مآورى

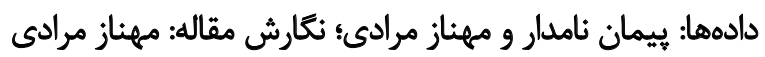

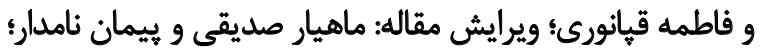
تنظيم نتايج: ليلى يكهفلاح و مهناز مرادى.

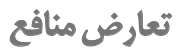

بنابر اظهار نويسندكان، اين مقاله تعارض منافع ندارد. 


\section{References}

[1] Zhu J, Zhong Z, Ji P, Li H, Li B, Pang J, et al. Clinicopathological characteristics of 8697 patients with COVID-19 in China: A meta-analysis. Fam Med Community Health. 2020; 8(2):e000406. [DOI:10.1136/fmch-2020-000406] [PMID] [PMCID]

[2] National Health Commission \& National Administration of Traditional Chinese Medicine. Diagnosis and treatment protocol for novel coronavirus pneumonia (Trial version 7). Chin Med J (Engl). 2020; 133(9):1087-95. [DOI:10.1097/ CM9.0000000000000819] [PMCID]

[3] Sheng G, Chen P, Wei Y, Yue H, Chu J, Zhao J, et al. Viral infection increases the risk of idiopathic pulmonary fibrosis: $A$ meta-analysis. Chest. 2020; 157(5):1175-87. [DOI:10.1016/j. chest.2019.10.032] [PMID] [PMCID]

[4] Wan S, Xiang Y, Fang W, Zheng Y, Li B, Hu Y, et al. Clinical features and treatment of COVID-19 patients in northeast Chongqing. J Med Virol. 2020; 92(7):797-806. [DOI:10.1002/ jmv.25783] [PMID] [PMCID]

[5] Pan L, Mu M, Yang P, Sun Y, Wang R, Yan J, et al. Clinical characteristics of COVID-19 patients with digestive symptoms in Hubei, China: A descriptive, cross-sectional, multicenter study. Am J Gastroenterol. 2020; 115(5):766-73. [DOI:10.14309/ ajg.0000000000000620] [PMID] [PMCID]

[6] Chung $M$, Bernheim A, Mei X, Zhang N, Huang M, Zeng X, et al. CT imaging features of 2019 novel Coronavirus (2019nCoV). Radiology. 2020; 295(1):202-7. [DOI:10.1148/radiol.2020200230] [PMID] [PMCID]

[7] Xie J, Covassin N, Fan Z, Singh P, Gao W, Li G, et al. Association between hypoxemia and mortality in patients with COVID-19. Mayo Clinic Proc. 2020; 95(6):1138-47. [DOI:10.1016/j.mayocp.2020.04.006] [PMID] [PMCID]

[8] Li K, Wu J, Wu F, Guo D, Chen L, Fang Z, et al. The clinical and chest $C T$ features associated with severe and critical COVID-19 pneumonia. Invest Radiol. 2020; 55(6):327-31. [DOI:10.1097/ RLI.0000000000000672] [PMID] [PMCID]

[9] Masetti C, Generali E, Colapietro F, Voza A, Cecconi M, Messina A, et al. High mortality in COVID-19 patients with mild respiratory disease. Eur J Clin Invest. 2020; 5(9):e13314. [DOI:10.1111/eci.13314] [PMID] [PMCID]

[10] Pan F, Ye T, Sun P, Gui S, Liang B, Li L, et al. Time course of lung changes at chest $C T$ during recovery from Coronavirus disease 2019 (COVID-19). Radiology. 2020; 295(3):715-21. [DOI:10.1148/radiol.2020200370] [PMID] [PMCID]

[11] Lal A, Mishra AK, Sahu KK, Noreldin M. Spontaneous pneumomediastinum: Rare complication of tracheomalacia. Arch Bronconeumol. 2019; 56(3):185-6. [DOI:10.1016/j.arbres.2019.09.017] [PMID]

[12] Brogna B, Bignardi E, Salvatore P, Alberigo M, Brogna C, Megliola $A$, et al. Unusual presentations of COVID-19 pneumonia on $\mathrm{CT}$ scans with spontaneous pneumomediastinum and loculated pneumothorax: A report of two cases and a review of the literature. Heart Lung. 2020; S0147-9563(20)30265-X. [DOI:10.1016/j.hrtlng.2020.06.005] [PMID] [PMCID]
[13] Anzueto A, Frutos-Vivar F, Esteban A, Alía I, Brochard L, Stewart $T$, et al. Incidence, risk factors and outcome of barotrauma in mechanically ventilated patients. Intensive Care Med. 2004; 30(4):612-9. [DOI:10.1007/s00134-004-2187-7] [PMID]

[14] Xu Z, Shi L, Wang Y, Zhang J, Huang L, Zhang C, et al. Pathological findings of COVID-19 associated with acute respiratory distress syndrome. Lancet Resp Med. 2020; 8(4):420-2. [DOI:10.1016/S2213-2600(20)30076-X]

[15] Aydin S, Oz G, Dumanli A, Balci A, Gencer A. A case of spontaneous pneumothorax in Covid-19 pneumonia. J Surg Res. 2020; 3(2):96-101. [DOI:10.26502/jsr.10020060]

[16] Chekkoth SM, Supreeth RN, Valsala N, Kumar P, Raja RS. Spontaneous pneumomediastinum in H1N1 infection: Uncommon complication of a common infection. J R Coll Physicians Edinb. 2019; 49(4):298-300. [DOI:10.4997/JRCPE.2019.409] [PMID]

[17] Bor C, Demirağ K, Uyar M, Cankayalı I, Moral AR. Recurrent spontaneous pneumothorax during the recovery phase of ARDS due to H1N1 infection. Balkan Med J. 2013; 30(1):123-5. [DOI:10.5152/balkanmedj.2012.086] [PMID] [PMCID]

[18] Ooi GC, Khong PL, Müller NL, Yiu WC, Zhou L, Ho JCM, et al. Severe acute respiratory syndrome: Temporal lung changes at thin-section CT in 30 patients. Radiology. 2004; 230(3):836-44. [DOI:10.1148/radiol.2303030853] [PMID]

[19] Han R, Huang L, Jiang H, Dong J, Peng H, Zhang D. Early clinical and CT manifestations of coronavirus disease 2019 (COVID-19) pneumonia. AJR Am J Roentgenol. 2020; 215(2):338-43. [DOI:10.2214/AJR.20.22961] [PMID]

[20] Zhou C, Gao C, Xie Y, Xu M. COVID-19 with spontaneous pneumomediastinum. Lancet Infect Dis. 2020; 20(4):510. [DOI:10.1016/S1473-3099(20)30156-0]

[21] Wang W, Gao R, Zheng Y, Jiang L. COVID-19 with spontaneous pneumothorax, pneumomediastinum and subcutaneous emphysema. J Travel Med. 2020; 27(5):taaa062. [DOI:10.1093/ jtm/taaa062] [PMID] [PMCID]

[22] Sun R, Liu H, Wang X. Mediastinal emphysema, giant bulla, and pneumothorax developed during the course of COVID-19 pneumonia. Korean J Radiol. 2020; 21(5):541-4. [DOI:10.3348/ kjr.2020.0180] [PMID] [PMCID]

[23] Sihoe ADL, Wong RHL, Lee ATH, Lau LS, Leung NYY, Law KI, et al. Severe acute respiratory syndrome complicated by spontaneous pneumothorax. Chest. 2004; 125(6):2345-51. [DOI:10.1378/chest.125.6.2345] [PMID] [PMCID]

[24] Xiang C, Wu G. SARS-CoV-2 pneumonia with subcutaneous emphysema, mediastinal emphysema, and pneumothorax A case report. Medicine (Baltimore). 2020; 99(20):e20208. [DOI:10.1097/MD.0000000000020208] [PMID] [PMCID]

[25] Flower L, Carter JPL, Lopez JR, Henry AM. Tension pneumothorax in a patient with COVID-19. BMJ Case Rep. 2020 13(5):e235861. [DOI:10.1136/bcr-2020-235861] [PMID] [PMCID] 
This Page Intentionally Left Blank 